\title{
Robust Time-domain Timing and Frequency Synchronization for OFDM Systems
}

\author{
Adegbenga B. Awoseyila, Christos Kasparis and Barry G. Evans
}

\begin{abstract}
A robust and efficient technique for frame/symbol timing and carrier frequency synchronization in OFDM systems is presented. It uses a preamble consisting of only one training symbol with two identical parts to achieve reliable timing and frequency accuracy in the time-domain, over a wide frequency estimation range which can be up to half of the signal sampling frequency. Also, it has a low complexity which is adaptive to the degree of channel distortion. Computer simulations in the Rayleigh fading ISI channel show that the proposed method achieves superior performance to existing techniques in terms of timing and frequency accuracy. Also, its operation in the time-domain helps to achieve faster synchronization convergence ${ }^{1}$.
\end{abstract}

Index Terms - OFDM, synchronization, cross-correlation, threshold detection.

\section{INTRODUCTION}

Orthogonal Frequency Division Multiplexing (OFDM) is a digital multi-carrier modulation technique, which uses several orthogonal sub-carriers to transmit/receive a high data rate signal [1]. It has become an increasingly popular scheme in modern digital communications and is already being applied in DAB, DVB-T, DVB-H, DVB-SH, WiFi, WiMAX and 3GPP LTE wireless standards. The primary advantage of OFDM over single-carrier transmissions is its robustness against frequency-selective fading in a multipath channel, thereby eliminating the need for complex time-domain equalization [1].

OFDM exhibits some tolerance to symbol timing errors when the cyclic prefix (CP) length is longer than the maximum channel delay spread [2]. However, an off-the-limit timing error (which positions the FFT window to include copies of either preceding or succeeding symbols) will result in inter-symbol interference (ISI) which degrades bit-errorrate (BER) performance [2].

On the other hand, OFDM is very sensitive to carrier frequency offsets in the received signal. Such frequency offsets may be caused by Doppler shifts and/or instabilities in the local oscillator (LO) and result in a loss of subcarrier orthogonality, thereby leading to inter-carrier interference (ICI). Consequently, it is usually required to reduce the frequency errors to a small fraction of the subcarrier spacing

\footnotetext{
${ }^{1}$ This work was supported in part by the EC FP6 SatNEx-II project (IST 027393).

A. B Awoseyila, C. Kasparis and B. G. Evans are with the Centre for Communication Systems Research, University of Surrey, Guildford, GU2 7XH, U.K. (e-mail: \{a.awoseyila, c.kasparis, b.evans\}@surrey.ac.uk).
}

[3]. It is noted that in practical system scenarios, the frequency offset can be many multiples of the subcarrier spacing due to the use of consumer-grade oscillators in the receiver [4]. Therefore, a wide frequency estimation range enables greater flexibility in terms of reducing the cost of OFDM receivers to mass-market consumers.

Various techniques have been proposed in the literature for combined timing and frequency synchronization in OFDM but in this paper, we focus on preamble-aided methods which can be applied to both burst-mode and continuous OFDM applications. Schmidl and Cox [5] proposed using the autocorrelation of a training symbol with two identical parts to estimate timing and fractional frequency offset in the timedomain. An additional training symbol is then used along with the first to determine the integer frequency offset in the frequency domain, within a range of $\pm N / 2$ subcarrier spacings, where $N$ is the FFT size. However, Schmidl's algorithm can yield timing estimates which are well beyond the ISI-free region and its integer frequency offset estimation becomes error-prone when the number of used subcarriers $N_{\text {use }}$ is significantly less than the FFT size $N$. Morelli's method [6] uses one training symbol with $J$ identical parts to achieve an improved frequency accuracy in the time-domain, but its estimation range of $\pm J / 2$ subcarrier spacings is not usually sufficient since each identical block must be longer than the channel delay spread in order to achieve reliable estimation. Kim's method [7] uses Schmidl's first training symbol to achieve integer frequency estimation in the frequency domain, within a range of $\pm N / 2$ subcarrier spacings. However, it experiences the same problem as Schmidl's method when $N_{\text {use }}$ is significantly less than $N$. In [8], Ren proposed a timedomain timing and frequency synchronization method based on a constant amplitude zero autocorrelation (CAZAC) training symbol with two identical parts, weighted by a binary pseudo-random noise (PN) sequence. It should be noted that Ren's timing algorithm is essentially the differential crosscorrelation of a randomized sequence, using a differential lag of $N / 2$. Although it yields a good timing performance in nonfading ISI channels, its accuracy degrades significantly under independent Rayleigh fading due to the weakness associated with differential cross-correlation. Moreover, its fractional frequency accuracy is poor due to the distortion of its preamble structure by the weighting PN sequence applied. Other preamble-aided techniques have been proposed for combined timing and frequency synchronization in [9]-[11] but they all suffer from a limited frequency estimation range and/or poor frequency accuracy. 
In this paper, we propose a novel technique that uses only one training symbol with a simple structure of two identical parts, to achieve robust, low-complexity and full-range timefrequency OFDM synchronization in the time-domain. It combines autocorrelation, restricted cross-correlation and threshold-based detection to achieve reliable synchronization accuracy in both AWGN and Rayleigh fading ISI channels. It is also able to achieve faster convergence than conventional techniques by completing its processing operations in the time-domain.

\section{SignAl Model}

The samples of an OFDM symbol at the output of the IFFT in the transmitter are given by:

$$
x(k)=\frac{1}{\sqrt{N}} \sum_{n=0}^{N_{\text {use }}-1} X_{n} e^{j 2 \pi k n / N} ; \quad k=0,1,2,3, \ldots . ., N-1
$$

where $N$ is the total number of subcarriers, with $N_{\text {use }}$ subcarriers being active. $X_{n}$ represents the data symbol transmitted on the nth subcarrier while $x(k)$ represents the symbol samples after IFFT processing, with each symbol consisting $N$ samples. However, the transmitted OFDM symbol can be denoted as $[x(N-G), \cdots, x(0), \cdots, x(N-1)]$ wherein the samples which precede $x(0)$ represent the cyclic prefix of length $G$ which is used to tolerate the intersymbol interference (ISI) resulting from the multipath channel delay spread.

Assuming sampling precision, the complex samples of the received signal from an ISI (wideband) channel can be represented as:

$$
r(k)=y(k-\theta) e^{j 2 \pi \varepsilon k / N}+\omega(k)
$$

where $\theta$ is the integer symbol timing offset measured in samples, $\varepsilon$ is carrier frequency offset normalized to the subcarrier spacing, $\omega(k)$ represents the zero-mean complex additive white Gaussian noise (AWGN) and

$$
y(k)=\sum_{l=0}^{L-1} h(l) x(k-l),
$$

where $x(k)$ represents the transmitted OFDM samples and $h(l)$ is the impulse response of the wideband channel whose memory order is $L-1$ samples.

\section{The Proposed Method}

The proposed method is a multi-stage process, aimed at maintaining low-complexity and fast synchronization convergence. Figure 1 shows a block diagram of typical synchronization stages in OFDM as implemented in [5],[7], while Figure 2 shows a block diagram that applies to the proposed method, wherein 'coarse timing' and 'fractional frequency' estimation are first achieved in similar fashion to Figure 1. These are then followed by the 'path timing checkpoint', 'joint path timing and integer frequency' and 'fine timing' estimation stages respectively. All the processing operations are performed using time-domain samples of the received signal, without the need for post-FFT subcarrier data recovery. Hence, the training symbol can be designed using optimized sequences in either the time-domain (TD) or frequency-domain (FD).

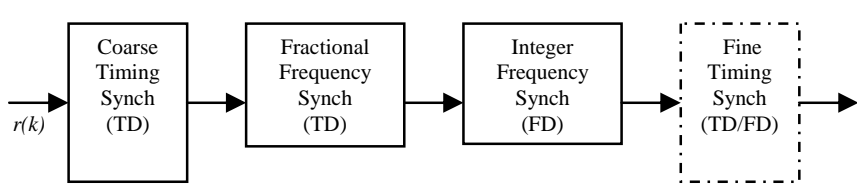

Fig. 1: Block diagram of typical synchronization stages in OFDM.

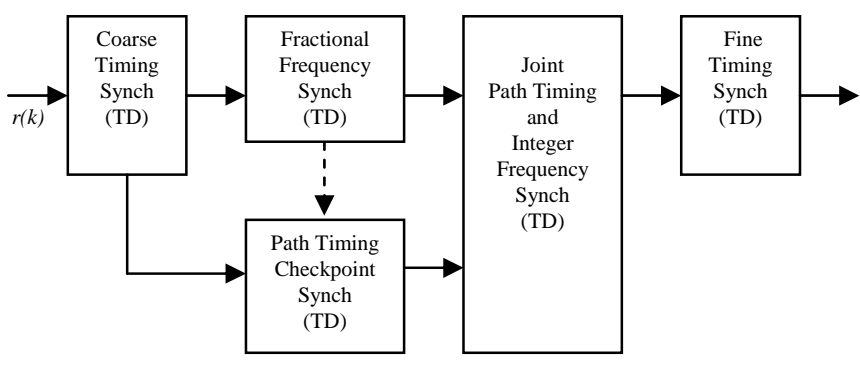

Fig. 2: Block diagram of synchronization stages used in the proposed method.

\section{A. Cross-correlation Detection in ISI channels}

In the AWGN channel, cross-correlation techniques (rather than autocorrelation) are widespread for frame timing detection and it has been shown that the maximum likelihood (ML) data-aided timing estimator in such channel is crosscorrelation based, under the assumption of negligible frequency offsets [12]. Similarly, it has been shown that the ML data-aided frequency estimator in the AWGN channel is also cross-correlation based under the assumption of ideal frame timing [13]. In this paper, we investigate crosscorrelation time-frequency detection in ISI (wideband) channels. It is first assumed that the transmitted signal $x(k)$ consists of an infinite random sequence which exists for positive and negative values of $k$, with a known part $s(k)$ (having sharp autocorrelation) such that $x(k)=S(k) ; \quad k=0,1,2 \cdots, N-1$. A cross-correlation between the received signal $r(k)$ from an ISI channel and the known PN sequence $S(k)$ can be derived as follows:

$$
P_{x}(d, \varepsilon)=\sum_{k=0}^{N-1} r(d+k, \varepsilon) S^{*}(k)
$$




$$
=\sum_{k=0}^{N-1}\left(\left(\sum_{l=0}^{L-1} h(l) X(d+k-\theta-l)\right) e^{j 2 \pi \varepsilon k / N}+\omega(k)\right) S^{*}(k),
$$

where $d$ represents the timing index.

Equation (5) can be re-arranged to show a coherent part $C$ (which can only occur at an arriving channel path) and a random part $\Gamma$ as follows:

$$
\begin{aligned}
& P_{x}(d, \varepsilon)=C(d=\theta+l, \varepsilon)+\Gamma(d \neq \theta+l, \varepsilon) \\
& C=\sum_{k=0}^{N-1} h(d-\theta)|S(k)|^{2} e^{j 2 \pi \varepsilon k / N} \\
& \Gamma=\sum_{k=0}^{N-1}\left(\left(\sum_{l \neq d-\theta} h(l) x(d+k-\theta-l)\right) S^{*}(k) e^{j 2 \pi \varepsilon k / N}+\omega(k) S^{*}(k)\right)
\end{aligned}
$$

where $(d-\theta)$ is the timing error.

It can be deduced from (6)-(8) that even in an ISI channel, cross-correlation is able to achieve joint timing and frequency detection due to the coherent summation in (7), produced at timing instants that correspond to the arrival of the known random sequence $S(k)$ via the channel taps i.e. $h(0), h(1), \ldots, h(L-1)$ in the absence of significant frequency offsets. The cross-correlation at all other timing instants will be lacking in the coherent part due to non-existent channel taps. Also, the presence of significant frequency offsets will destroy such coherence. For instance, assuming $S(k)$ has constant amplitude, the coherent summation in (7) reduces to zero for integer frequency offsets since the sum of a sinusoid over an integer number of full cycles is zero. Therefore a known random sequence (having sharp autocorrelation) can be used to achieve joint timing and integer frequency detection via the sharp detection properties of crosscorrelation.

Deriving from the central limit theorem (CLT) and assuming that complete frequency synchronization has been achieved, the cross-correlation $P_{x}(d, 0)$ at all other timing instants apart from those corresponding to the channel paths (i.e. $\forall d \neq d_{\text {path }}$, where $d_{\text {path }} \in\{\theta, \theta+1, \cdots, \theta+L-1\}$ ) can be taken to be a zero-mean complex Gaussian variable. Using the same CLT principle and assuming a timing instant that corresponds to the arrival of a channel path and that reliable fractional frequency synchronization has been achieved, the cross-correlation $P_{x}\left(d_{\text {path }}, \varepsilon_{i}\right)$ at all other integer frequency corrections apart from that corresponding to the actual integer frequency offset correction can be taken to be a zero-mean complex Gaussian variable. This implies that the absolute value of the cross-correlation at the non-coherent instants in either the timing axis (i.e. $\left.\left|P_{x}\left(d_{N C}, 0\right)\right|\right)$ or the frequency axis (i.e. $\left|P_{x}\left(d_{\text {path }}, \varepsilon_{i N C}\right)\right|$ ) will follow a Rayleigh distribution whose probability density function (PDF) and expected value are given as:

$$
\begin{aligned}
& p_{R}\left(\left|P_{x}\left(\beta_{N C}\right)\right|\right)=\frac{\left|P_{x}\left(\beta_{N C}\right)\right|}{\sigma^{2}} e^{-\frac{\left|P_{x}\left(\beta_{N C}\right)\right|^{2}}{2 \sigma^{2}}}, \\
& \mathrm{E}\left(\left|P_{x}\left(\beta_{N C}\right)\right|\right)=\sigma \sqrt{\pi / 2},
\end{aligned}
$$

where $\mathrm{E}(\cdot)$ is the expected value operator and $\sigma^{2}$ is the variance of either the real or imaginary components of $P_{x}\left(\beta_{N C}\right) . \quad \beta_{N C}$ is a non-coherent instant which represents either $d_{N C}$ in the timing axis or $\varepsilon_{i N C}$ in the frequency axis.

Assuming that sufficient observations are available, the noise parameter $\sigma$ can be estimated using the low-complexity algorithm shown below. Alternatively, it can be estimated using the ML estimator described in [14].

$$
\hat{\sigma}\left(\beta_{N C}\right)=\sqrt{2 / \pi} \cdot\left(\operatorname{mean}_{\beta_{N C}}\left\{\left|P_{x}\left(\beta_{N C}\right)\right|\right\}\right),
$$

Deriving from the Rayleigh cumulative distribution function (CDF) as shown in (12)-(14), a threshold $\delta$ can be chosen in accordance with a desired probability that $\left|P_{x}\left(\beta_{N C}\right)\right|$ does not exceed such threshold, wherein the reverse case is the probability of false alarm: $\mathrm{P}_{F A}$. Assuming that $\mathrm{P}_{F A}<<1$ for a set of $\lambda$ Rayleigh variables, the probability of false detection $P_{F D}$ (i.e. the probability that any of the noncoherent variables will exceed the chosen threshold) will increase arithmetically relative to one instant and can be approximated by (15).

$$
\begin{aligned}
& F(\delta)=\mathrm{P}\left(\left|P_{x}\left(\beta_{N C}\right)\right|<\delta\right)=1-e^{\left(-\delta^{2} / 2 \sigma^{2}\right)}, \\
& \mathrm{P}\left(\left|P_{x}\left(\beta_{N C}\right)\right|>\delta\right)=\mathrm{P}_{F A}=e^{\left(-\delta^{2} / 2 \sigma^{2}\right)}, \\
& \delta=\sqrt{-\ln \left(\mathrm{P}_{F A}\right) 2 \sigma^{2}}, \\
& \mathrm{P}_{F D} \approx \lambda \mathrm{P}_{F A} .
\end{aligned}
$$

Consequently, a timing or frequency threshold can be chosen based on the principles discussed in this section, such that the absolute value of the cross-correlation at the coherent timing and integer frequency instants exceed the chosen threshold while that of all other timing and/or integer frequency instants fall below such threshold with high 
probability. This threshold crossing detection technique applied to both timing and frequency axis is one of the key components of our proposed method, such that reliable and low-complexity time-domain synchronization in OFDM systems is achieved.

\section{B. A Simple Training Symbol}

A training symbol having two identical parts in the timedomain is chosen for the proposed method. The structure as shown in equation (16) is also implemented in [5], [8], [15]. Its autocorrelation property helps to achieve reliable and lowcomplexity preamble detection, coarse timing and fractional frequency estimation while its uniquely combined auto- and cross-correlation properties are used in our proposed method to achieve enhanced time-frequency synchronization performance.

$$
S=\left[\begin{array}{ll}
A_{N / 2} & A_{N / 2}
\end{array}\right]
$$

where $A_{N / 2}$ is a random sequence consisting of $N / 2$ samples in the time-domain.

The training symbol can be generated in the frequencydomain by mapping a QPSK PN sequence to the evennumbered subcarriers, as specified in [5]. Alternatively, it can be generated in the time-domain via direct repetition, using a suitable PN sequence. In contrast to Ren's method, there is no multiplication of the training symbol with a weighting PN sequence in the proposed method.

\section{Coarse Timing and Fractional Frequency}

Firstly, coarse timing estimation is achieved in similar fashion to [15] wherein a low-complexity autocorrelation of the received signal samples is computed as shown in (17) and a coarse timing estimate derived accordingly as shown in (18) and (19). Fractional frequency offset estimation is then achieved by taking the phase of the autocorrelation at the coarse timing estimate, which can be pre-advanced towards the middle of the cyclic prefix zone (i.e. $\theta_{c}-0.5 G$ ) in order to improve accuracy. Such pre-advancement is not applicable where a cyclic prefix is not used. The fractional frequency offset is corrected in the stored received signal samples as shown in (21). Consequently, the residual frequency offset becomes an integer multiple of 2 since the estimator in (20) has an estimation range of \pm 1 subcarrier spacing. This implies that the integer search window is halved.

$$
\begin{aligned}
& P_{S c h}(d)=\sum_{k=0}^{N / 2-1} r^{*}(d+k) r(d+k+N / 2) \\
& M_{c}(d)=\frac{1}{G+1} \sum_{k=0}^{G}\left|P_{S c h}(d-k)\right|^{2}, \\
& \hat{\theta}_{c}=\underset{d}{\arg \max }\left\{M_{c}(d)\right\},
\end{aligned}
$$

$$
\begin{aligned}
& \hat{\varepsilon}_{f}=\frac{1}{\pi} \angle\left\{P_{S c h}\left(\hat{\theta}_{c}-0.5 G\right)\right\}, \\
& r_{\text {cor }}(k)=r(k) e^{-j 2 \pi k \hat{\varepsilon}_{f} / N},
\end{aligned}
$$

where $P_{S c h}(d)$ is Schmidl's autocorrelation [5] which is integrated in (18) over the length of the CP in order to eliminate its uncertainty plateau and achieve a coarse timing metric $M_{c}(d)$ whose peak indicates the coarse timing estimate $\hat{\theta}_{c} \cdot \hat{\varepsilon}_{f}$ is the fractional frequency offset estimate and $r_{\text {cor }}(k)$ is the fractional-frequency-corrected received signal sample.

\section{Path Timing Checkpoints}

After coarse timing and fractional frequency synchronization are achieved, restricted differential crosscorrelation between the received signal samples and the known preamble $S(k)$ is computed using a restricted set of timing instants as indicated by a timing filter window derived from the coarse timing metric $M_{c}(d)$.

Differential cross-correlation is used in order to cope with large frequency offsets (i.e. $\varepsilon \geq 1$ ) as coherent crosscorrelation will fail under this condition [11], [16]. However, differential cross-correlation is not as reliable as coherent cross-correlation for timing detection purposes because the extra terms it incorporates increase the overall noise variance. Nevertheless, differential cross-correlation techniques produce sharper detection properties than autocorrelation [16] and in an ISI environment, differential cross-correlation between a received preamble and its known version (i.e. a random sequence having sharp autocorrelation) can be used to detect a set of likely timing instants corresponding to the arriving channel paths.

Figure 1 shows an example of a single-lag differential cross-correlation of the fractional-frequency-corrected received signal as implemented using (22)-(24) under ideal channel conditions, wherein there is a major peak in the middle corresponding to a full-symbol pattern match and two minor peaks at half-symbol timing error corresponding to a half-symbol pattern match. The minor peaks result from the symmetric structure of the preamble and constitute a major hindrance to using such preamble in a noisy channel since any of these peaks could instantaneously have the highest value.

$$
\begin{aligned}
& U(d, k)=r_{c o r}(d+k) S^{*}(k) ; \quad k \in\{0, N-1\} \\
& P_{d x}(d)=\sum_{k=0}^{N-2} U^{*}(d, k) U(d, k+1), \\
& M_{d x}(d)=\left|P_{d x}(d)\right|^{2}
\end{aligned}
$$


where $U(d, k)$ represents the samples of the fractionalfrequency-corrected received signal $r_{\text {cor }}$ at timing point $d$ multiplied by their corresponding complex conjugate in the known preamble $S(k) . P_{d x}(d)$ is the differential crosscorrelation and $M_{d x}(d)$ is the differential cross-correlation metric.

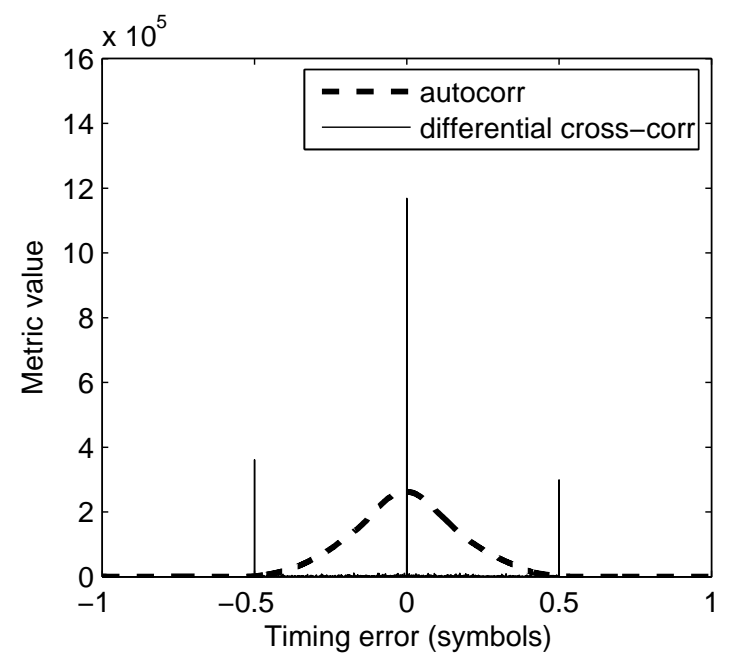

Fig. 1 Autocorrelation and differential cross-correlation metrics for the proposed method in an ideal channel; $N=1024, G=72$

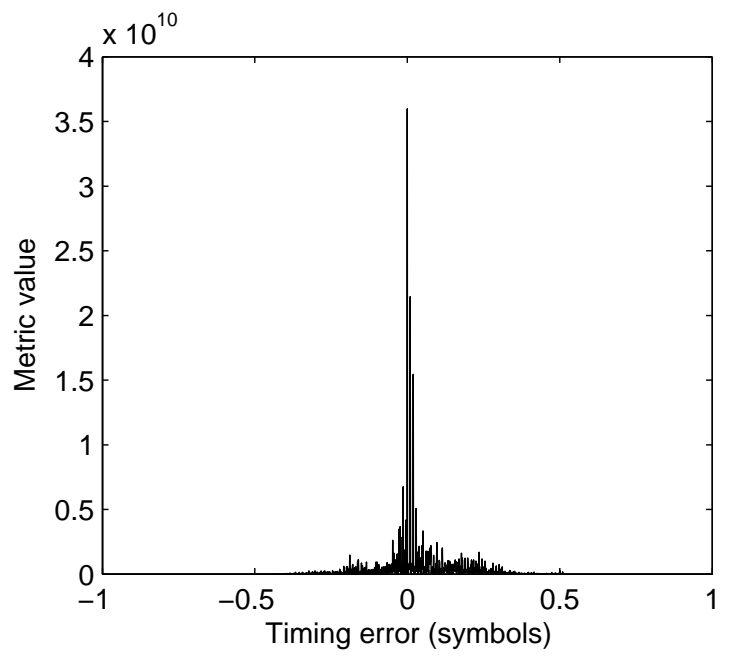

Fig. 2 Filtered differential cross-correlation metric for the proposed method in a Rayleigh fading ISI channel; $N=1024, G=72$

As discussed in [15], the autocorrelation metric of the training symbol (shown in Figure 1) can be used to filter the unwanted minor peaks. One way to achieve this is to simply multiply both metrics together. This multiplication can be restricted to a timing window of one symbol length $N$ or less, symmetric around the coarse timing estimate $\hat{\theta}_{c}$ in order to reduce complexity. An alternative approach which avoids multiplication is to use the autocorrelation peak value to define a timing window in terms of all timing points whose autocorrelation exceeds a pre-determined fraction of the peak value. This timing window is then used to restrict the differential cross-correlation accordingly. The filtering helps to determine a set of likely timing instants which correspond to the arriving channel paths. Figure 2 shows the resulting timing metric achieved by such filtering process in the test Rayleigh fading ISI channel under no additive noise.

The processing operations within the differential crosscorrelation stage are summarized as follows:

$$
\begin{gathered}
M_{\text {check }}(d)=\left|P_{d x}(d)\right|^{2} \cdot M_{c}(d) ; \\
d \in\left\{\hat{\theta}_{c}-N / 2, \hat{\theta}_{c}+N / 2\right\} \\
\hat{d}_{\text {check }}=\underset{d}{\arg \max }\left\{M_{\text {check }}(d)\right\}
\end{gathered}
$$

where $M_{\text {check }}(d)$ is the filtered timing checkpoint metric computed for a restricted set of timing points and $d$ is chosen in (25) to ensure that all relevant timing points that could be the ideal timing are tracked. $d_{\text {check }}$ represents a generic timing checkpoint while $\hat{d}_{\text {check }}$ is the operational timing checkpoint which is estimated in (26) after excluding all previously determined timing checkpoints from $M_{\text {check }}(d)$.

\section{E. Joint Path Timing and Integer Frequency}

The next stage is an enhanced algorithm which can operate in the two dimensions (2D) of timing and frequency to jointly determine a strong channel path timing and the integer frequency offset. This estimator combines the strength of coherent cross-correlation for timing and integer frequency detection as discussed earlier in section III-A.

The timing checkpoints are determined by sorting the filtered timing-checkpoint metric $\left(M_{\text {check }}(d)\right)$ in descending order of strength. For each timing checkpoint starting from the strongest, the corresponding one-dimensional vector of $N$ fractional-frequency-corrected samples undergoes coherent cross-correlation with the known preamble $S(k)$ over all possibilities of integer frequency offset correction. This approach is similar to Ren's frequency algorithm [8]. However, the integer frequency metric can be computed more efficiently by the use of FFT processing as shown in (27). It should be noted that the integer frequency metric can only give accurate results if the timing estimate corresponds to a strong arriving channel path as discussed in III-A.

$$
\begin{gathered}
I\left(\hat{d}_{\text {check }}, i\right)=\left|\operatorname{FFT}\left\{U\left(\hat{d}_{\text {check }}, k\right)\right\}\right| ; \\
k \in\{0, N-1\}
\end{gathered}
$$

where $I\left(\hat{d}_{\text {check }}, i\right)$ is the $1 \mathrm{D}$ integer frequency offset metric, determined at the operational timing checkpoint $\hat{d}_{\text {check }}$, using 
the fast Fourier transform (FFT).

The proposed 1D/2D time-frequency estimator is adaptive in terms of complexity as it uses both threshold crossing (TC) and maximum value (MAX) detection criteria [16]. The TC criterion is implemented over the $1 \mathrm{D}$ integer frequency offset metric at each timing checkpoint while the MAX criterion is implemented over the complete 2D time-frequency metric.

For each timing checkpoint starting from the strongest, the algorithm derives a 1D integer frequency offset metric according to (27). The peak value of the $1 \mathrm{D}$ metric at a timing checkpoint is compared with a threshold $\delta_{F}$. If this threshold is exceeded, a successful estimation is declared for both channel path timing and integer frequency at that peak instant. Otherwise the next timing checkpoint is used and the process repeated until there is either success or the complete 2D timefrequency metric is formed and its maximum value determined as the successful estimate. This is implemented as follows:

$$
\begin{aligned}
& \hat{\varepsilon}_{i}=\underset{i}{\arg \max }\left\{I\left(d_{\text {check }}, i\right)\right\} \\
& \hat{d}_{\text {path }}=\underset{d_{\text {check }}}{\operatorname{argmax}}\left\{I\left(d_{\text {check }}, i\right)\right\}
\end{aligned}
$$

where $\hat{\varepsilon}_{i}$ is the integer frequency offset estimate and $\hat{d}_{\text {path }}$ is the timing estimate which corresponds to a strong arriving channel path. $I\left(d_{\text {check }}, i\right)$ is either the $1 \mathrm{D}$ integer frequency offset metric at an operational timing checkpoint where the TC criterion indicates successful estimation or the complete 2D time-frequency metric wherein all timing checkpoints have been tested and the MAX criterion used.

By using the threshold criterion, the proposed algorithm is able exclude timing instants which do not correspond to a strong arriving channel path and thereby overcome the weakness associated with differential cross-correlation under channel noise, in contrast to Ren's method.

The threshold $\delta_{F}$ is determined based on the statistics of the Rayleigh distribution as explained in section III-A. The probability of false detection in the frequency axis $P_{F D_{F}}$ should be chosen low enough to avoid a synchronization failure since a missed detection can still be recovered via the MAX criterion of the 2D metric.

$$
\begin{aligned}
& \delta_{F}=\sqrt{-\ln \left(\mathrm{P}_{F D_{F}} / \lambda_{F}\right) 2 \hat{\sigma}_{F}{ }^{2}} \\
& \hat{\sigma}_{F}=\sqrt{2 / \pi} \cdot\left(\underset{i}{\operatorname{mean}}\left\{I\left(\hat{d}_{\text {check }}, i\right)\right\}\right) ; \quad i \neq \hat{\varepsilon}_{i}
\end{aligned}
$$

where $\lambda_{F}$ is the search window used for integer frequency detection.
The maximum number of timing checkpoints $\left(n_{\max }\right)$ needed for reliable joint time-frequency estimation is inversely proportional to the FFT size and the SNR since these parameters directly affect the accuracy of cross-correlation. Nonetheless, for a small FFT size, an increase in $n_{\max }$ is balanced out by the reduction in computational complexity since $N$ is small. Ultimately, the pre-determined design value to be used depends on the tolerable processing delay for synchronization. Based on extensive simulations, the following range is recommended, without loss of generality.

$$
1 \leq n_{\max } \leq 64 ; \quad N \geq 64
$$

It is noted that under favourable channel conditions, the first timing checkpoint is usually sufficient to achieve reliable synchronization. After estimating the integer frequency offset, compensation is made for the total frequency offset in the received signal as follows:

$$
r_{\text {corT }}(k)=r_{\text {cor }}(k) e^{-j 2 \pi k \hat{\varepsilon}_{i} / N}
$$

where $r_{\text {corT }}(k)$ is the total-frequency-corrected received signal sample.

\section{F. Fine Timing}

In order to adjust the timing estimate to the ideal start of frame which corresponds to the first arriving channel path (rather than any strong arriving channel path $\hat{d}_{\text {path }}$ as determined by the joint time-frequency algorithm), a modified version of the timing adjustment technique proposed in [15] is used, since $\hat{d}_{\text {path }}$ is already known. The processing operations within the final coherent cross-correlation stage are summarized as follows:

$$
\begin{aligned}
& P_{x}(d)=\sum_{k=0}^{N-1} r_{\text {corT }}(d+k) S^{*}(k) \text {; } \\
& d \in\left\{\hat{d}_{p a t h}-N / 2+\lambda_{T}+1, \hat{d}_{\text {path }}\right\} \\
& \hat{\theta}_{F F T}=\arg f i r s t\left\{\left|P_{x}(d)\right|>\delta_{T}\right\} ; \\
& d \in\left\{\hat{d}_{p a t h}-\lambda_{T}, \hat{d}_{p a t h}\right\} \\
& \delta_{T}=\sqrt{-\ln \left(\mathrm{P}_{F D_{T}} / \lambda_{T}\right) 2 \hat{\sigma}_{T}^{2}} \\
& \hat{\sigma}_{T}=\sqrt{2 / \pi} \cdot\left(\underset{d}{\operatorname{mean}}\left\{\left|P_{x}(d)\right|\right\}\right) ; \\
& d \in\left\{\hat{d}_{p a t h}-N / 2+\lambda_{T}+1, \hat{d}_{p a t h}-\lambda_{T}-1\right\},
\end{aligned}
$$

where $d$ is chosen in (34) to ensure that all relevant timing points that could be the ideal timing are tracked while 
maintaining low-complexity. $d$ is chosen in (35) such as to track the first arriving path ( $\hat{\theta}_{F F T}$ ) rather than any strong arriving channel path $\left(\hat{d}_{\text {path }}\right)$, wherein all channel paths are expected to be received within $\lambda_{T}+1$ samples: ( $L-1 \leq \lambda_{T} \leq G$ ). In (37), $d$ is chosen such as to exclude major and minor coherent peaks and their multipath, in order to calculate the timing threshold $\delta_{T}$ using an estimate of the noise parameter $\sigma_{T} \cdot \mathrm{P}_{F D_{T}}$ is probability of false detection in the timing axis, which is chosen to achieve a reliable timing accuracy without aggravating the occurrence of missed detection. The first arriving channel path (or any other one) is expected to have a correlation which is greater than $\delta_{T}$ with high probability. The threshold $\delta_{T}$ is therefore used to detect the ideal timing $\hat{\theta}_{F F T}$.

\section{COMPUTER Simulations}

Extensive computer simulations were performed in order to assess the performance of the proposed method in comparison with existing methods for combined timing and frequency synchronization in OFDM. QPSK sub-carrier modulation is used with a random normalized frequency offset uniformly distributed in the range: $-N / 2<\varepsilon<N / 2$. The search window for timing adjustment is set to $\lambda_{T}=G$ for the proposed method and the training symbol is generated in the frequency domain as specified in [5]. The probability of false detection is set as $\mathrm{P}_{F D_{F}}=\mathrm{P}_{F D_{T}}=10^{-6}$ for the proposed joint time-frequency and timing adjustment algorithms.

The OFDM parameters are chosen similar to the 3GPP LTE specifications for a $10 \mathrm{MHz}$ bandwidth, with $N=1024$, $N_{\text {use }}=600, G=72$ and a sampling frequency $F_{s}=15.36 \mathrm{Msymb} / \mathrm{s}$. The ISI channel parameters are chosen similar to the test channel specified in Kim's paper [7] with a carrier frequency $F_{c}=5 \mathrm{GHz}$ and a mobile speed of $150 \mathrm{~km} / \mathrm{h}$, wherein the channel consists of $M=6$ paths (i.e. $m=0,1,2, \ldots, 5$ ) having path delays of $\tau_{m}=10 \mathrm{~m}$ samples and an exponential power profile with average tap power $\rho_{m}=\exp \left(-\tau_{m} / 30\right) / \sum_{m=0}^{M-1} \exp \left(-\tau_{m} / 30\right)$. Each path undergoes independent Rayleigh fading.

For the results of Figure 3 and 5, the number of used subcarriers is set to $N_{u s e}=N$, mobile speed to zero and the power-delay-profile of the test ISI channel is implemented with only additive noise, in order to compare with a similar approach in [8],[18], wherein the channel taps do not undergo independent Rayleigh fading.

In Figure 3, the proposed method achieves an ideal timing accuracy with zero timing mean-square-error (MSE) while the timing MSE of Ren's method is fractional at moderate SNR and shown to be significantly better than Schmidl's method. However, when independent time-variant Rayleigh fading is introduced in Figure 4, the performance of Ren's method is significantly degraded as compared to Schmidl's method. This is due to the weakness associated with differential crosscorrelation in the presence of channel noise. The proposed method maintains its superior performance by achieving a near-ideal timing MSE which corresponds to detecting the first arriving channel path, unless it is significantly faded.

In terms of frequency estimation performance, Figure 5 shows that the proposed method, Schmidl's and Kim's method achieve a reliable frequency accuracy in a non-fading ISI channel while Ren's method has a poor fractional accuracy due to the distortion of the identical blocks in its training symbol by the weighting PN sequence applied. The CramerRao lower bound (CRLB) for single frequency estimation using $N$ samples [13] is also shown for comparison purposes.

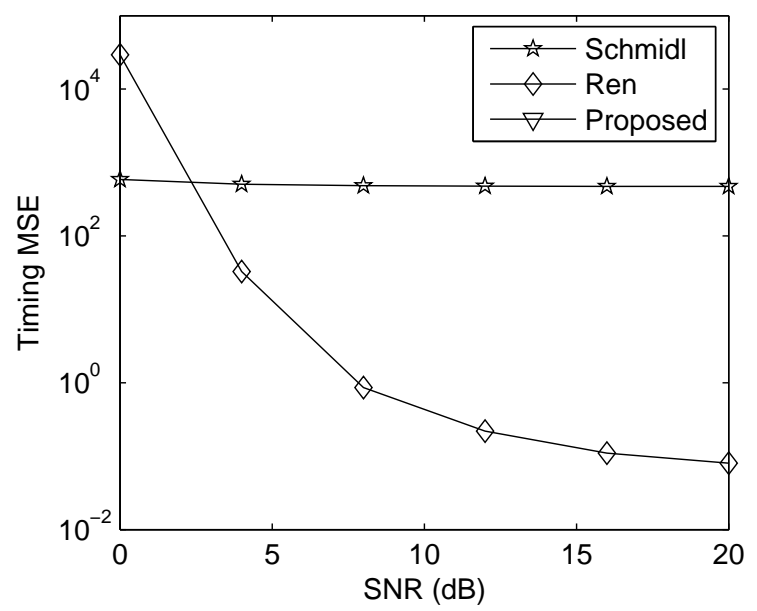

Fig. 3 Timing MSE performance in a non-fading ISI channel with additive noise; $N=1024, N_{\text {use }}=1024, G=72$

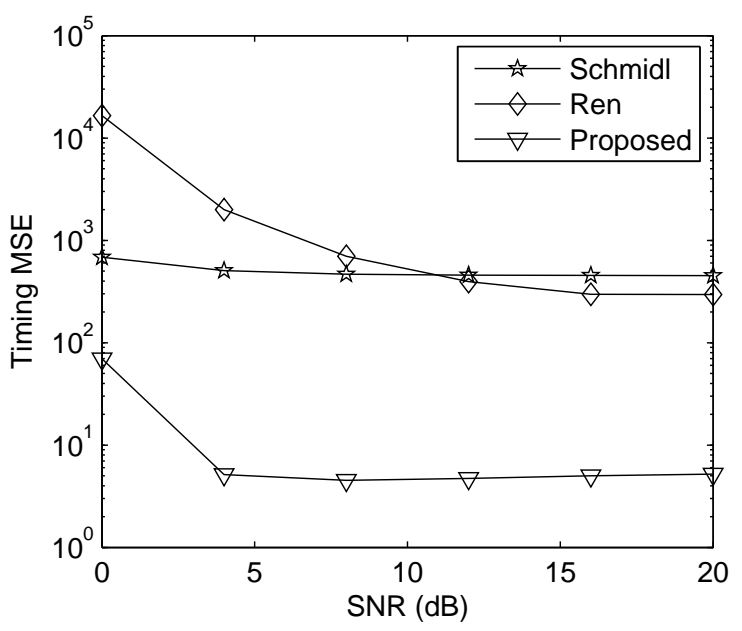

Fig. 4 Timing MSE performance in a Rayleigh fading ISI channel with additive noise; $N=1024, N_{\text {use }}=600, G=72$

The results of Figure 6 show the performance of the frequency estimators under independent time-variant Rayleigh fading and a low $N_{u s e} / N$ ratio. The proposed method is shown to achieve a reliable frequency accuracy in contrast to the 
existing methods which experience large estimation variance due to integer frequency errors. This problem is caused in Ren's method by the poor performance of its timing algorithm while it is caused in Schmidl's and Kim's methods by their operation in the frequency domain under a low $N_{\text {use }} / N$ ratio.

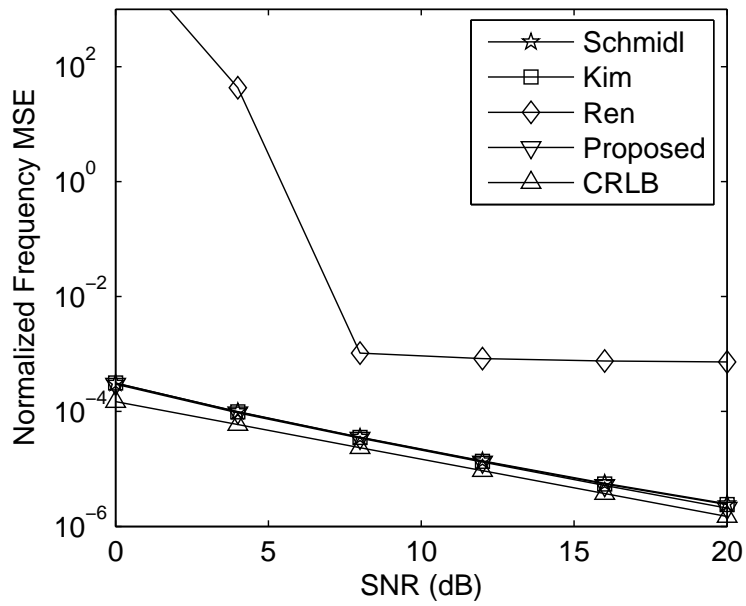

Fig. 5 Frequency MSE performance in a non-fading ISI channel with additive noise; $N=1024, N_{\text {use }}=1024, G=72$

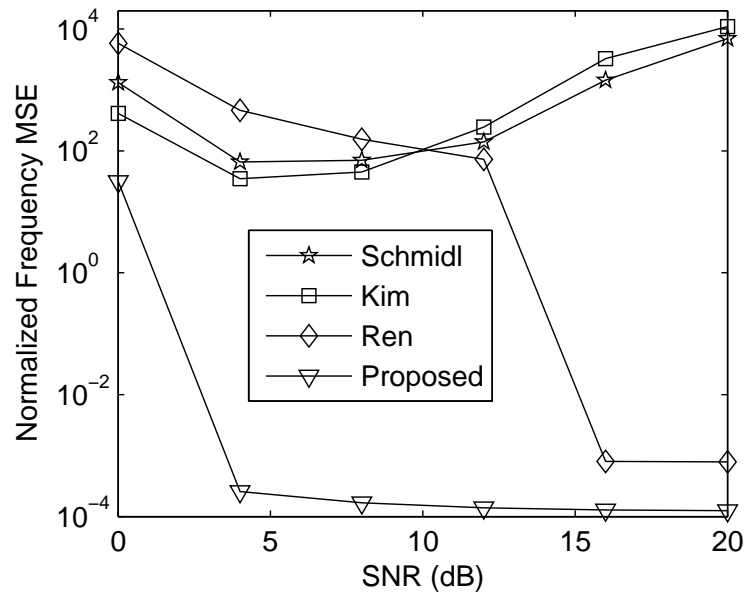

Fig. 6 Frequency MSE performance in a Rayleigh fading ISI channel with additive noise; $N=1024, N_{u s e}=600, G=72$

\section{CONCLUSIONS}

A novel technique which uses only one training symbol with a simple structure of two identical parts to achieve robust, low-complexity and full-range time-frequency synchronization in OFDM systems is proposed. Computer simulations in the Rayleigh fading ISI channel shows that the proposed technique achieves a superior performance to the existing methods, wherein its timing MSE approaches the ideal value of zero while its wide-range integer frequency estimation is reliable even when the $N_{\text {use }} / N$ ratio is low, in contrast to the existing methods. Also, it has a low and adaptive complexity as it makes use of effective threshold detection and efficient FFT processing. Furthermore, it achieves fast convergence by completing all its processing operations in the time-domain.

\section{REFERENCES}

[1] R. van Nee and R. Prasad, OFDM for Wireless Multimedia Communications, Artech House, 1999.

[2] M. Morelli, C.-C. J. Kuo, and M.-O. Pun, "Synchronization Techniques for Orthogonal Frequency Division Multiple Access (OFDMA): A Tutorial Review,” Proc. IEEE, vol. 95, no. 7, pp. 1394-1427, Jul. 2007.

[3] T. Pollet, M. van Bladel, and M. Moeneclaey, "BER Sensitivity of OFDM Systems to Carrier Frequency Offset and Weiner Phase Noise," IEEE Trans. Commun., vol. 43, pt.1, pp. 191-193, Feb.-Apr. 1995.

[4] N. Balamurali and D. Jalihal, "Robust timing and frequency synchronization algorithm for IEEE 802.16 OFDM systems,” Intl. Conf. Signal Process. Commun. (SPCOM), pp. 234-238, 2004.

[5] T. Schmidl and D. C. Cox, "Robust frequency and timing synchronization for OFDM,” IEEE Trans. Commun., vol. 45, no. 12, pp. 1613 - 1621, Dec. 1997.

[6] M. Morelli and U. Mengali, “An improved frequency offset estimator for OFDM systems,” IEEE Commun. Lett., vol. 3, no. 3, pp. 239-241, Mar. 1999.

[7] Y. H. Kim, I. Song, S. Yoon, and S. Park, "An efficient frequency offset estimator for OFDM systems and its performance characteristics,” IEEE Trans. Vehicular Tech., vol. 50, no. 5, pp. 1307-1312, Sep. 2001.

[8] G. Ren, Y. Chang, H. Zhang, and H. Zhang, "Synchronization methods based on a new constant envelope preamble for OFDM systems," IEEE Trans. Broadcast., vol. 51, no. 1, pp.139-143, Mar. 2005.

[9] H. Minn, V. K. Bhargava, and K. B. Letaief, "A robust timing and frequency synchronization for OFDM systems," IEEE Trans. W. Commun., vol. 2, no. 4, pp. 822-839, Jul. 2003.

[10] K. Shi and E. Serpedin, "Coarse frame and carrier synchronization of OFDM systems: A new metric and comparison," IEEE Trans. W. Commun., vol. 3, no. 4, pp. 1271-1284, Jul. 2004.

[11] C. Kasparis and B. G. Evans, "A cross-correlation approach for improved timing estimation in OFDM broadcasting systems," 24th AIAA Intl. Commun. Satell. Sys. Conf. (ICSSC), pp. 1039-1048, 2006.

[12] J. L. Massey, “Optimum Frame Synchronization," IEEE Trans. Commun., vol. 20, no. 2, Apr. 1972.

[13] M. Morelli and U. Mengali, "Carrier Frequency Estimation for Transmissions over Selective Channels,” IEEE Trans. Commun., vol. 48, no. 9, pp. 1580-1589, Sep. 2000.

[14] M. Peritsky, "Statistical Estimation of Mean Signal Strength in a Rayleigh-Fading Environment,” IEEE Trans. Commun., vol. 21, no. 11, Nov. 1973.

[15] A. B. Awoseyila, C. Kasparis, and B. G. Evans, "Improved preambleaided timing estimation for OFDM systems," IEEE Commun. Lett., vol. 12, no. 11, pp. 825-827, Nov. 2008.

[16] Kim et al, "Enhanced frame synchronization for DVB-S2 system under a large frequency offset," Wireless Commun. Networking Conf. (WCNC) 2007, pp. 1184 - 1188, Mar. 2007.

[17] 3GPP TS 36.211, 3rd Generation Partnership Project; Technical Specification Group Radio Access Network; Evolved Universal Terrestrial Radio Access (E-UTRA); Physical Channels and Modulation (Release 8), 2008.

[18] Y. Kang, S. Kim, D. Ahn, and H. Lee, "Timing estimation for OFDM systems by using a correlation sequence of preamble," IEEE Trans. Consumer Electron., vol. 54, no. 4, pp. 1600-1608, Nov. 2008.

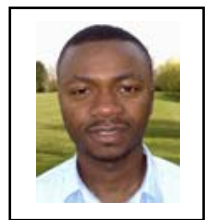

Adegbenga B. Awoseyila received the B.Sc. in Electronics and Electrical Engineering (1st class honours) from the Obafemi Awolowo University (O.A.U) Ile-Ife, Nigeria in 1998, M.Sc. in Mobile and Satellite Communications (with Distinction) and Ph.D. in Electronic Engineering from the University of Surrey Guildford, U.K. in 2005 and 2008 respectively. He is currently working as a Research Fellow at the Centre for Communication systems Research (CCSR), University of Surrey, U.K. His research interests cover signal processing techniques for wireless systems (including estimation 
and synchronization) and physical/link layer techniques for satellite-mobile communications.

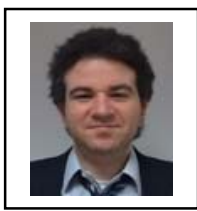

Christos Kasparis received the B.Eng. in Electronics and Telecoms (1st class honours) from the University of Birmingham U.K. in 2000, M.Sc. in Communications Systems and Signal Processing (with Distinction) and Ph.D. in Iterative Signal Processing Techniques for Radio-Communication Systems from the University of Bristol U.K. in 2001 and 2005 respectively. He has worked as a Research Fellow at the Centre for Communication systems Research (CCSR), University of Surrey, Guildford, U.K (2005-2008) and is currently working as a Researcher at the Mitsubishi Electric Research Centre Europe (U.K.). His Research interests cover signal processing techniques for radio-wireless systems: multi-user signal detection, space-time processing, OFDM PAPR reduction, FEC decoding, synchronization and estimation.

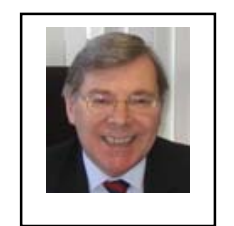

Barry G. Evans is director of the Centre for Communications Systems Research (CCSR), University of Surrey, Guildford, U.K., where he is a Professor and also Pro-Vice Chancellor for Research and Enterprise. He is editor of the International Journal of Satellite Communications and Networking and a well-known international consultant in mobile and satellite communications, having researched for over 30 years in the field. He is author of over 600 publications in the literature and of several books on telecommunications and satellites. He has served on several IEE professional groups and ITU-R study groups and for twenty years has run the IEE vacation school on satellite communications at Surrey. In 1990 he was elected to a fellowship of the Royal Society of Arts and Manufacturing Sciences and in 1991 as a Fellow of the Royal Academy of Engineering. 